\title{
Desempenho de sementes peletizadas de alface em função do material cimentante e da temperatura de secagem dos péletes.
}

\author{
João Bosco C. Silva ${ }^{1}$; Paulo E.C. Santos ${ }^{2}$; Warley Marcos Nascimento ${ }^{1}$. \\ 'Embrapa Hortaliças, C. Postal 218, 70.359-970 Brasília - DF, E-mail: jbosco@enph.embrapa.br ${ }^{2}$ Graduando de agronomia, UnB.
}

\section{RESUMO}

Sementes de alface da cultivar Grand Rapids Nacional foram peletizadas, utilizando-se como material de enchimento, a mistura de microcelulose e areia fina em volumes iguais e, como material cimentante, diferentes volumes de suspensão aquosa de bentonita e de acetato de polivinila, em cinco porporções (\%): $100+0 ; 75+25 ; 50+50$; $25+75$; e $0+100$. Os cimentantes foram aplicados em separado, sendo a bentonita aplicada na primeira camada de recobrimento das sementes. Após a peletização, as sementes foram submetidas à secagem sob temperaturas de 15 e $36^{\circ} \mathrm{C}$, e avaliadas quanto à germinação e emergência das plântulas. As combinações de cimentantes utilizadas na peletização e a temperatura de secagem não afetaram significativamente a porcentagem final de germinação das sementes em gerbox. Na avaliação da emergência de plântulas em bandejas, apenas duas formulações de péletes apresentaram menor taxa em relação ao tratamento com desempenho máximo, indicando que as diferenças obtidas não foram devidas às diferenças na formulação do recobrimento e nem à temperatura de secagem. Independentemente da temperatura de secagem, todas as formulações utilizadas na peletização causaram redução na velocidade de germinação. Entretanto, quando semeadas em substrato orgânico e cultivadas em casa de vegetação, as sementes revestidas com várias formulações apresentaram índices de velocidade de emergência semelhantes aos das sementes não peletizadas. Neste ambiente, as plântulas oriundas de sementes peletizadas apresentaram crescimento normal, sem diferenças significativas com a testemunha, em relação à produção de matéria seca da parte aérea e das raízes, avaliadas aos 20 dias após a semeadura.

Palavras-chave: Lactuca sativa L., peletização, estabelecimento de plântulas, vigor.

\begin{abstract}
Performance of pelleted lettuce seeds in response to glue material and temperature during the drying of the pellets.

Seeds of lettuce cv. Grand Rapids Nacional were pelleted using as a filler, a mixture of sand and microcellulose in equal volume parts, and as a glue, different volumes of water suspension of bentonite and polyvinyl acetate at five proportions (\%): $100+0$; $75+25 ; 50+50 ; 25+75 ;$ and $0+100$ applied apart, betonite being applied in the first coat layer. After pelleting, seeds were dried at 15 or $36^{\circ} \mathrm{C}$ and evaluated for germination and seedling emergence. Seed pelleting compound and drying temperature did not affect total germination. Only two pellet formulations showed reduced rate of seedling emergence indicating that differences were not due to the coat formulation and drying process. Independently of seed pellet formulation and dry temperature, the germination of pelleted seed delayed in relation to raw seeds. When sowed in organic substrate and placed under greenhouse conditions, pelleted seeds showed similar germination rate to raw seeds. In this environment, seedlings from pelleted seeds showed normal growth. The fresh and dry matter were similar to those from raw seeds at 20 days after sowing.
\end{abstract}

Keywords: Lactuca sativa L., pelleting, coat, seedling establishment, vigor.

(Aceito para publicação em 09 de janeiro de 2.002)

\begin{abstract}
A peletização consiste no revestimento de sementes com sucessivas camadas de material seco e inerte, dando a elas o formato arredondado, maior massa e acabamento liso, o que facilita a distribuição e o manuseio das sementes, especialmente aquelas muito pequenas, pilosas, rugosas ou deformadas. As sementes de alface (Lactuca sativa, L.) caracterizam-se pelo pequeno tamanho, pouco peso e formato irregular, o que dificulta a sua individualização e distribuição, tanto no processo manual quanto mecânico de semeadura. A utilização de sementes peletizadas reduz os custos de produção de mudas por diminuir o consumo de sementes, diminui o ser-
\end{abstract}

viço de distribuição manual de sementes, facilita a mecanização da semeadura e pode eliminar a prática do desbaste de plantas excedentes. Soma-se a isto, a possibilidade de incorporação de nutrientes, reguladores de crescimento e outros agroquímicos durante o processo de peletização, podendo constituir melhorias na sanidade das sementes e no estabelecimento das plântulas. Para algumas espécies, o menor consumo de sementes pode viabilizar a utilização de semente de melhor qualidade genética, especialmente as sementes híbridas.

Embora a técnica de peletização tenha sido desenvolvida há vários anos, as informações referentes à composição dos materiais empregados e à confecção dos péletes são pouco difundidas, uma vez que esta técnica permanece inacessível junto às empresas de sementes e as companhias processadoras dos péletes.

O processo consiste basicamente em aplicar camadas sucessivas de um material sólido inerte sobre as sementes em constante movimento dentro de uma betoneira, alternando a aplicação do material de enchimento com a pulverização de um cimentante solúvel em água (Silva, 1997; Silva \& Nakagawa, 1998a).

Para escolha de material a ser utilizado na peletização, incluindo os de enchimento, os adesivos e os de acabamen- 
to, deve-se levar em conta a sua influencia, dentre outros aspectos, na rigidez do pélete, na absorção de água e na troca gasosa entre a semente e o ambiente externo ao pélete. Todos estes aspectos afetam diretamente a germinação das sementes, causando geralmente, redução na velocidade de germinação e do crescimento das plântulas (Sachs et al., 1981; Sachs et al., 1982; Silva \& Márton, 1992; Silva et al., 1992).

Silva, (1997) e Silva \& Nakagawa, (1998a; 1998b; 1998c; 1998d), propuseram diferentes métodos para avaliação de materiais e verificaram diferenças marcantes quanto ao tempo de dissolução e a resistência física dos péletes, fatores estes que influenciam a absorção de água e a troca gasosa, afetando assim a germinação das sementes.

Em avaliações preliminares para seleção de material (dados não publicados), definiu-se a areia fina, microcelulose, bentonita e acetato de polivinila como as melhores opções de ingredientes para formulação da camada de peletização. A areia e a microcelulose são utilizadas como material para enchimento. A bentonita e a cola à base de acetato de polivinila (cola PVA) são utilizadas como cimentante, tendo a bentonita a função de formar o núcleo dos péletes, por ser uma argila que agrega levemente as partículas utilizadas como enchimento. A cola PVA é utilizada para cimentar a camada de acabamento, por ter maior capacidade de agregação das partículas, dando a rigidez necessária aos péletes. No entanto, os adesivos formam uma solução de alta viscosidade, o que dificulta a troca gasosa entre a semente e o ambiente externo ao pélete, sendo portanto interessante minimizar o uso de cola PVA.

O processo de peletização implica na aplicação de um volume relativamente grande de água, utilizada como veículo para pulverização da suspensão de cimentantes. Após o processamento, a umidade contida na camada de peletização deve ser retirada imediatamente, evitando assim a absorção de água pela semente. Porém o uso de calor durante essa secagem pode causar dormência secundária às sementes (Popinigis, 1985).

O objetivo do trabalho foi avaliar a viabilidade e o desenvolvimento de plântulas de alface em resposta a diferentes proporções de bentonita e cola PVA utilizadas na peletização, bem como avaliar o efeito da temperatura de secagem após o processamento.

\section{MATERIAL E MÉTODOS}

\section{Peletização}

Sementes de alface do tipo crespa, da cultivar Grand Rapids Nacional, foram peletizadas em uma betoneira adaptada e instalada na Embrapa Hortaliças, seguindo metodologia descrita por Silva \& Nakagawa (1998a). Utilizou-se, como material para enchimento, uma mistura de areia fina e microcelulose, em volumes iguais, peneirada aos poucos e aplicada alternadamente com a pulverização de cimentante. Como material cimentante, variou-se a proporção dos volumes de solução aquosa bentonita (BT), na diluição de $80 \mathrm{~g} / \mathrm{L}$ e de cola à base de acetato de polivinila (PVA), diluída a 25\% (v/v), nas seguintes proporções (\%) de BT + PVA: $100+0 ; 75+25 ; 50+50 ; 25+75 ; 0+100$. Cada ingrediente cimentante foi aplicado separadamente por pulverização dirigida à massa circulante dentro da betoneira, sendo o PVA aplicado na fase de acabamento. Ou seja, os núcleos dos péletes foram construídos com cimentante bentonita e a camada externa com PVA.

Após a peletização, as sementes foram divididas em duas amostras e secadas em estufas sob temperaturas constantes de 15 e de $36^{\circ} \mathrm{C}$, por 24 horas, constituindo um fatorial $5 \times 2$, mais uma testemunha constituída das sementes nuas.

\section{Germinação}

Quatro repetições de 50 péletes ou sementes nuas foram distribuídas sobre duas folhas de papel de germinação, umedecidas com água destilada, e incubadas em caixas gerbox, mantidas em germinadores a $20^{\circ} \mathrm{C}$, sob luz. Foram realizadas contagens diárias das sementes que emitiram raízes primárias. A contagem final de germinação foi realizada aos sete dias, conforme as prescrições estabelecidas pelas Regras de Análise de Sementes (Brasil, 1992). Utilizando os dados de contagem diária de germinação calculou-se o índice de velocidade de germinação por meio da fórmula proposta por Maguirre (1962).

\section{Emergência de plântulas e produ-} ção de mudas

Três repetições de 128 sementes foram semeadas em bandejas de poliestireno de 128 células, contendo substrato orgânico comercial da marca Plantmax (Eucatex) e mantidas em casa de vegetação. Foi realizada a contagem diária da emergência das plântulas e, aos 20 dias, efetuou-se a colheita das mudas para determinação das massas de matéria fresca e seca, tanto da parte aérea como das raízes. Para a determinação da massa de matéria seca utilizouse uma estufa de circulação forçada, a $60^{\circ} \mathrm{C}$ por 24 horas.

\section{Rapidez e eficiência de plantio}

Determinou-se o tempo médio gasto por operários treinados para semeadura manual em bandejas de 128 células, comparando sementes nuas e peletizadas. Outra avaliação foi realizada com uma semeadora mecânica, dotada de mecanismo de sucção das sementes por agulhas, determinando-se a porcentagem de células sem sementes bem como a porcentagem de células com mais de uma semente.

A análise dos dados foi realizada inicialmente no modelo de fatorial excluindo-se o tratamento testemunha, visando analisar os efeitos interativos. Não havendo interação significativa, fez-se a análise com todos os tratamentos, discriminando-os por meio do teste de Dunnet.

\section{RESULTADOS E DISCUSSÃO}

\section{Germinação}

A porcentagem de germinação obtida em laboratório não foi afetada pelas diversas composições de cimentantes utilizados na peletização das sementes (Tabela 1), não se observando também diferenças na germinação quanto à temperatura de secagem dos péletes. Entretanto, as sementes nuas emitiram raízes primárias mais rapidamente que todos os tipos de sementes peletizadas, sendo que entre as composições de péletes e entre as temperaturas de secagem não houve diferenças significativas. Este atraso na germinação é comum em sementes peletizadas conforme resultados de diversos estudos (Silva, 1997; Sachs et al., 1981; Roos \& Moore III, $1981 \mathrm{e}$ trabalhos citados por Tonkin, 1984). 


\section{Emergência de plântulas}

Todos os péletes apresentaram porcentagem de emergência semelhantes à obtida com sementes nuas; apenas dois tipos de péletes apresentaram menor taxa de emergência de plântulas em relação ao tratamento com desempenho máximo $\left(100 \% \mathrm{BT} / 36^{\circ} \mathrm{C}\right)$, sendo um dos tipos confeccionado com a formulação de $50 \%$ de cola à base de PVA e secagem a $15^{\circ} \mathrm{C}$ e outro com $25 \%$ de cola PVA e secagem a $36^{\circ} \mathrm{C}$ (Tabela 2). Estes resultados sugerem que as diferenças na emergência de plantulas não podem ser relacionadas com a formulação da camada de recobrimento e nem com a temperatura de secagem.

Quanto à velocidade de emergência das plântulas, o comportamento das sementes peletizadas foi mais aproximado das sementes não cobertas, quando se comparam estes dados com os obtidos na avaliação da velocidade de germinação, avaliada em laboratório.

O retardamento observado na emergência das plântulas obtidas com sementes peletizadas, não afetou significativamente, a qualidade das mudas de alface produzidas em bandejas sob condições de casa de vegetação (Tabela 2). Aos 20 dias após a semeadura, as

Tabela 1. Germinação de sementes nuas e de diversos tipos de péletes de semente de alface 'Grand Rapids Nacional' incubadas sob luz, a 20 C. Brasília, Embrapa Hortaliças, 1999.

\begin{tabular}{lccc}
\hline $\begin{array}{c}\text { Composição de } \\
\text { cimentante }\end{array}$ & $\begin{array}{c}\text { Temperatura } \\
\text { de secagem } \\
\left({ }^{\circ} \mathbf{C}\right)\end{array}$ & $\begin{array}{c}\text { Germinação } \\
(\%)\end{array}$ & $\begin{array}{c}\text { Índice de } \\
\text { velocidade }\end{array}$ \\
\hline $100 \%$ BT & 36 & $96,5 \mathrm{a}$ & $21,3 \mathrm{~b}$ \\
$75 \%$ BT + 25\% PVA & 36 & $96,0 \mathrm{a}$ & $20,5 \mathrm{~b}$ \\
$50 \%$ BT + 50\% PVA & 36 & $96,0 \mathrm{a}$ & $19,7 \mathrm{~b}$ \\
$25 \%$ BT + 75\% PVA & 36 & $95,0 \mathrm{a}$ & $19,3 \mathrm{~b}$ \\
$100 \%$ PVA & 36 & $96,0 \mathrm{a}$ & $21,3 \mathrm{~b}$ \\
$100 \%$ BT & 15 & $98,0 \mathrm{a}$ & $22,0 \mathrm{~b}$ \\
$75 \%$ BT + 25\% PVA & 15 & $94,0 \mathrm{a}$ & $20,2 \mathrm{~b}$ \\
$50 \%$ BT + 50\% PVA & 15 & $91,5 \mathrm{a}$ & $19,3 \mathrm{~b}$ \\
$25 \%$ BT + 75\% PVA & 15 & $95,5 \mathrm{a}$ & $19,9 \mathrm{~b}$ \\
$100 \%$ PVA & 15 & $93,5 \mathrm{a}$ & $19,8 \mathrm{~b}$ \\
Sementes nuas (controle) & - & $97,5 \mathrm{a}$ & $25,4 \mathrm{a}$ \\
\hline CV (\%) & & 3,3 & 5,7 \\
\hline DMS & & 7,73 & 2,87 \\
\hline
\end{tabular}

Valores seguidos pela mesma letra não diferem entre si pelo teste de Tukey na comparação entre péletes e pelo teste Dunnett, na comparação com a testemunha.

$\mathrm{BT}=$ Bentonita; $\mathrm{PVA}=$ Cola à base de acetato de polivinila

plântulas não apresentaram diferenças significativas de massa de matéria fresca e seca, tanto da parte aérea quanto das raízes, quando se comparam as plântulas originadas de sementes nuas com as originadas dos demais tratamen- tos, inclusive para as temperaturas de secagem e diferentes tipos de péletes. Entretanto, percebe-se pelo alto coeficiente de variação, que houve desuniformidade no crescimento das plântulas.

Tabela 2. Emergência e desenvolvimento de plântulas oriundas de diversos tipos de péletes de sementes de alface 'Grand Rapids Nacional', em casa de vegetação. Brasília, Embrapa Hortaliças, 1999.

\begin{tabular}{|c|c|c|c|c|c|}
\hline \multirow{2}{*}{ Composição dos péletes } & \multirow{2}{*}{$\begin{array}{l}\text { Temperatura de } \\
\text { secagem }\left({ }^{\circ} \mathrm{C}\right)\end{array}$} & \multirow{2}{*}{ Emergência (\%) } & \multirow{2}{*}{$\begin{array}{l}\text { Índice de } \\
\text { velocidade }\end{array}$} & \multicolumn{2}{|c|}{ Matéria seca $(\mathrm{mg})^{*}$} \\
\hline & & & & Parte aérea & Raízes \\
\hline $100 \%$ BT & 36 & 96,6 a & $34,7 \mathrm{abc}$ & $260 \mathrm{a}$ & $160 \mathrm{a}$ \\
\hline $75 \%$ BT + 25\% PVA & 36 & $90,1 \quad b$ & $33,0 \quad c$ & $371 \mathrm{a}$ & $130 \mathrm{a}$ \\
\hline $50 \%$ BT + 50\% PVA & 36 & $91,4 a b$ & 33,4 bc & 352 a & 108 a \\
\hline $25 \%$ BT $+75 \%$ PVA & 36 & $94,5 a b$ & $33,8 \mathrm{abc}$ & 367 a & 108 a \\
\hline $100 \%$ PVA & 36 & $92,2 \mathrm{ab}$ & $34,5 \mathrm{abc}$ & 378 a & $160 \mathrm{a}$ \\
\hline $100 \%$ BT & 15 & $94,5 a b$ & $36,4 a b$ & 363 a & $147 \mathrm{a}$ \\
\hline $75 \%$ BT + 25\% PVA & 15 & $94,0 \mathrm{ab}$ & $35,1 \mathrm{abc}$ & 395 a & $143 a$ \\
\hline $50 \%$ BT + 50\% PVA & 15 & $90,1 \quad b$ & $32,5 \quad c$ & $312 \mathrm{a}$ & 118 a \\
\hline $25 \%$ BT + 75\% PVA & 15 & $91,7 \mathrm{ab}$ & $34,0 \mathrm{abc}$ & $303 a$ & 178 a \\
\hline $100 \%$ PVA & 15 & $91,4 a b$ & $34,4 \mathrm{abc}$ & $491 \mathrm{a}$ & $182 \mathrm{a}$ \\
\hline Sementes nuas (controle) & - & $95,6 a b$ & 36,6 a & $404 a$ & $135 a$ \\
\hline $\mathrm{CV}$ & & 2,3 & 3,2 & 17,8 & 36,3 \\
\hline DMS & & 6,17 & 3,18 & 190 & 105 \\
\hline
\end{tabular}

*Média de 20 plântulas.

Valores seguidos pela mesma letra não diferem entre si pelo teste de Tukey na comparação entre péletes e pelo teste Dunnett, na comparação com a testemunha.

$\mathrm{BT}=$ Bentonita; $\mathrm{PVA}=$ Cola à base de acetato de polivinila 


\section{Rapidez e eficiência de plantio}

Quanto à avaliação da facilidade de semeio, verificou-se que para semear manualmente as sementes peletizadas, os operários gastaram o tempo médio de 2:12 minutos para semear uma bandeja de 128 células, e gastaram 3:42 minutos para semear sementes nuas, $o$ que corresponde a um gasto a mais de $68 \%$ de mão-de-obra. Observou-se ainda, naquelas bandejas semeadas com sementes nuas, que algumas células continham mais de uma semente, o que é um aspecto negativo à produção de mudas, pois além de um maior gasto de sementes, há a necessidade de se realizar, posteriormente, o desbaste naquelas células com mais de uma plântula.

As sementes peletizadas foram facilmente semeadas em semeadora mecânica, e não se observou nenhum tipo de dano ou quebra dos péletes, causado pela semeadora.

\section{AGRADECIMENTOS}

Os autores agradecem à Horti-mudas - Xavier \& Baliza Ltda., por ter ce- dido operários e equipamentos para realização das avaliações de rapidez e eficiência de plantio das sementes.

\section{LITERATURA CITADA}

BRASIL. Ministério da Agricultura e Reforma Agrária. Regras para análise de sementes. Brasília, Secretaria Nacional de Defesa Agropecuária. 1992. 365 p.

MAGUIRRE, J.D. Speed of germination-aid in selection and evaluation for seedling emergence and vigor. Crop Science, v. 2, p. 176-7,1962.

NASCIMENTO, W.M.; CALIARI, M.F.; MARCOS FILHO, J. Influência da quantidade de água no substrato na germinação de sementes peletizadas de alface, beterraba e tomate. Horticultura Brasileira, Brasília, v. 7, n. 1, p. 68, 1989. (resumo 169)

POPINIGIS, F. Fisiologia da semente. Brasília, s. ed., 289 p., 1985

ROOS, E.E.; MOORE III, F.D. Effect of seed coating on performance of lettuce seeds in greenhouse soil tests. Journal of the American Society for Horticultural Science, v. 106, p. 385389, 1981.

SACHS, M.; CANTLIFFE, D.J.; NELL, T.A. Germination behavior of sand-coated sweet pepper seed. Journal of the American Society for Horticultural Science, v. 107, p. 412-416, 1982.

SACHS, M; CANTLIFFE, D.J.; NELL, T.A. Germination of clay-coated sweet pepper seeds. Journal of the American Society for Horticultural Science, v. 106, p. 385-389, 1981.
SILVA, J.B.C.; MÁRTON, L. Adaptation of pelletization techniques of seeds in Brazil. IN: International Scientific Conference. [ON] The application and utilization of the agricultural scientific results in developing countries, 2, 1992, Godollo, Hungria. [papers....]. Godollo: University of agriculture, tropical and subtropical agriculture department, 1992, p. 286-289.

SILVA, J.B.C.; MÁRTON, L.; NASCIMENTO, W.M. Peletização de sementes com calcário. Horticultura Brasileira, Brasília, v. 10, n. 1, p. 69, 1992. (resumo 129)

SILVA, J.B.C. Avaliação de métodos e materiais para peletização de sementes. Botucatu: UNESP, 1997. 127 p.(Tese doutorado)

SILVA, J.B.C.; NAKAGAWA, J. Confecção e avaliação de péletes de sementes de alface, Horticultura Brasileira, Brasília, v. 16, n. 2, p. 151-158, 1998a.

SILVA, J.B.C.; NAKAGAWA, J. Metodologia para avaliação da resistência de péletes. Horticultura Brasileira, Brasília, v. 16, n. 2, p. 118-122, 1998b.

SILVA, J.B.C.; NAKAGAWA, J. Metodologia para avaliação de materiais cimentantes para peletização de sementes. Horticultura Brasileira, Brasília, v. 16, n. 1, p. 31-37, 1998c.

SILVA, J.B.C.; NAKAGAWA, J. Métodos para avaliação de materiais de enchimento utilizados na peletização de sementes. Horticultura Brasileira, Brasília, v. 16, n. 1, p. 44-49, 1998d.

TONKIN, J.H.B. Pelleting and other pre-sowing treatments. In: THOMPSON, J.R. (Ed.) Advances in research and technology of seeds, part 9. Wageningen: ISTA, p. 95-97, 1984. 